\title{
Editorial
}

\section{Advanced Topics in Fractional Dynamics}

\section{Dumitru Baleanu, ${ }^{1}$ H. M. Srivastava, ${ }^{2}$ Varsha Daftardar-Gejji, ${ }^{3}$ Changpin $\mathrm{Li}^{4}{ }^{4}$ and J. A. Tenreiro Machado ${ }^{5}$}

${ }^{1}$ Department of Mathematics and Computer Sciences, Cankaya University, Ankara, Turkey

${ }^{2}$ Department of Mathematics and Statistics, University of Victoria, Victoria, BC, Canada V8W 3R4

${ }^{3}$ Department of Mathematics, University of Pune, Pune 411007, India

${ }^{4}$ Department of Mathematics, Shanghai University, Shanghai 200444, China

${ }^{5}$ Department of Electrical Engineering, Institute of Engineering, Polytechnic of Porto, Rua Dr. Antonio Bernardino de Almeida 431, 4200-072 Porto, Portugal

Correspondence should be addressed to Dumitru Baleanu; dumitru@cankaya.edu.tr

Received 4 November 2013; Accepted 4 November 2013

Copyright ( 2013 Dumitru Baleanu et al. This is an open access article distributed under the Creative Commons Attribution License, which permits unrestricted use, distribution, and reproduction in any medium, provided the original work is properly cited.

Fractional order differentiation consists in the generalisation of classical integer differentiation to real or complex orders.

During the last decades, fractional differentiation has drawn increasing attention in the study of so-called anomalous social and physical behaviours, where scaling power law of fractional order appears universal as an empirical description of such complex phenomena.

The goal of this special issue is to address the latest developments in the area of fractional calculus application in dynamical systems.

The special issue received 38 publications and 24 of high quality papers were accepted. The papers of this special issue have a large variety of interesting and relevant subjects, namely, fractional partial differential equations, numerical algorithms, chaos, complexity and fractional calculus, fractals, and power law.

\author{
Dumitru Baleanu \\ H. M. Srivastava \\ Varsha Daftardar-Gejji \\ Changpin Li \\ J. A. Tenreiro Machado
}




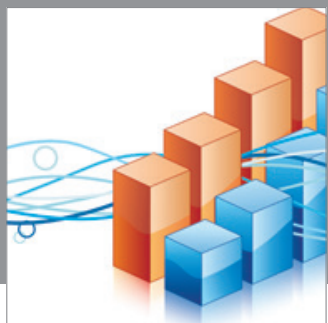

Advances in

Operations Research

mansans

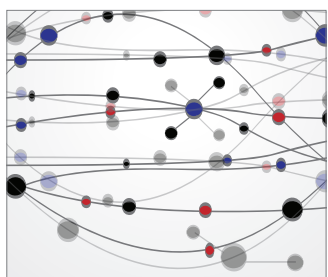

The Scientific World Journal
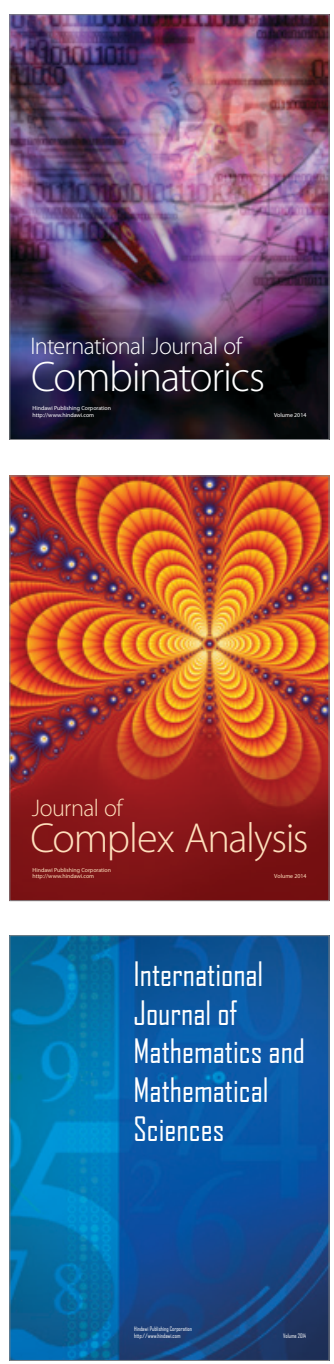
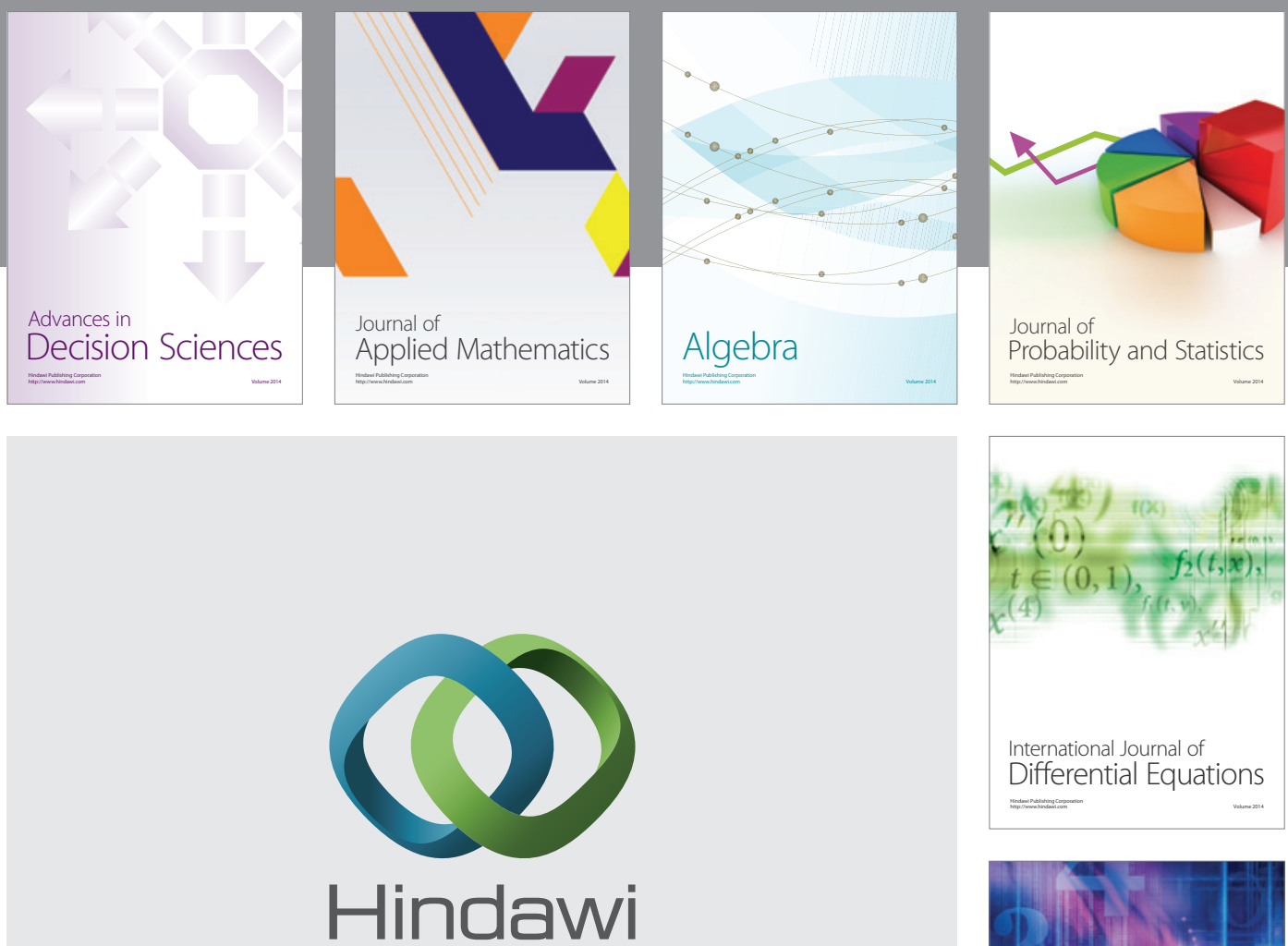

Submit your manuscripts at http://www.hindawi.com
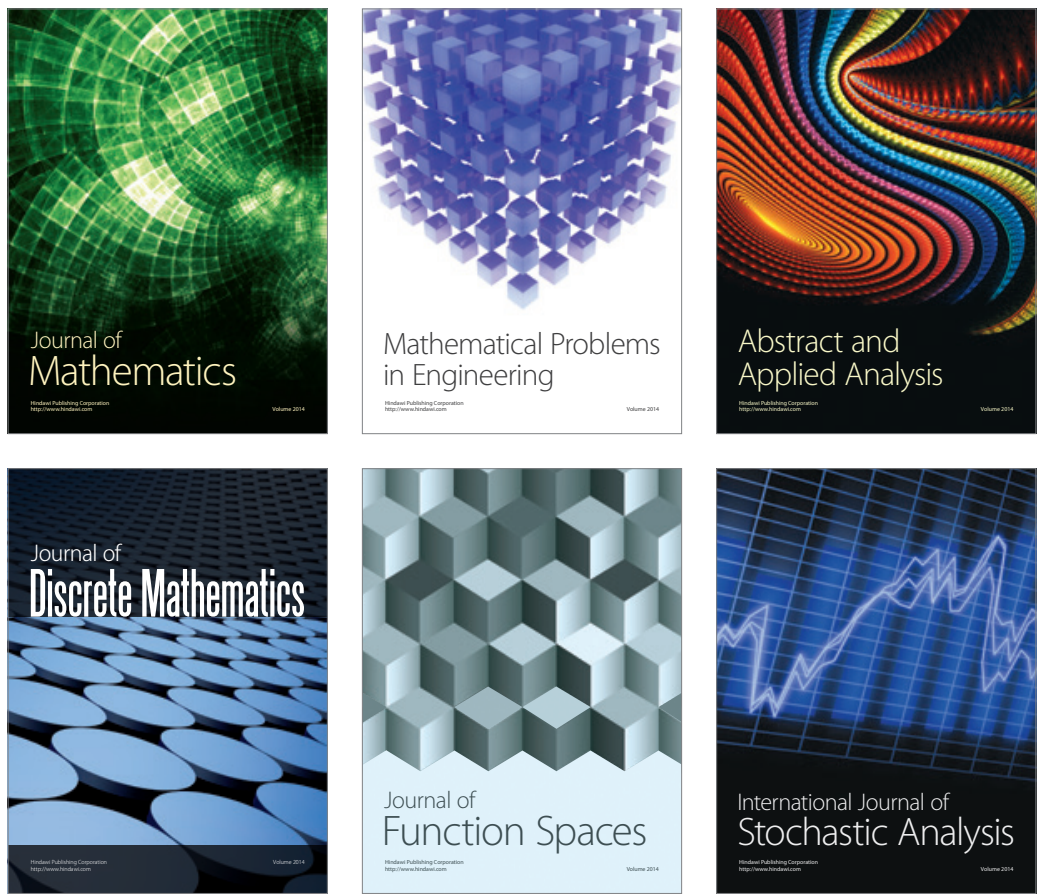

Journal of

Function Spaces

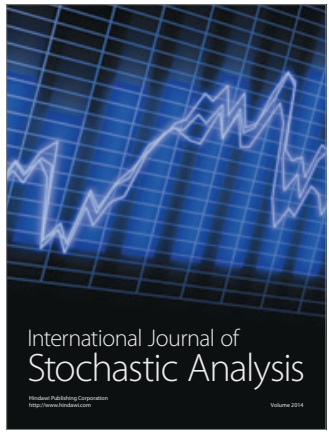

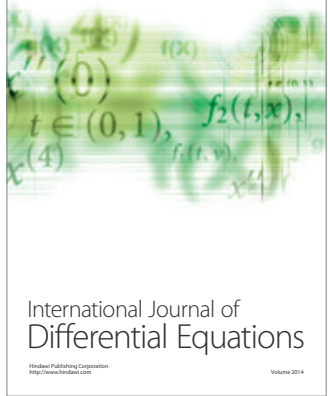
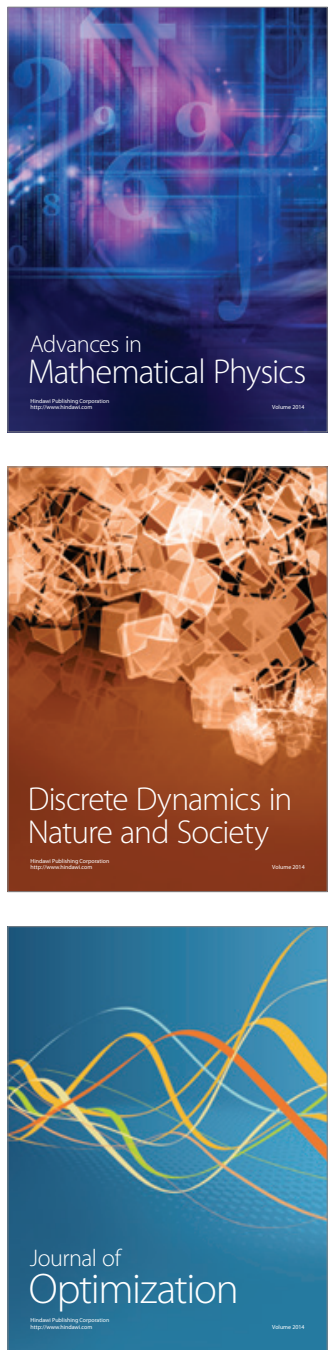